\title{
Iterative Surface Construction for Blind Deflectometry
}

\author{
Wenchuan Zhao ${ }^{\mathrm{a}, \mathrm{b}}$, Logan R. Graves ${ }^{\mathrm{b}}$, Run Huang $^{\mathrm{b}}$, Weihong Song ${ }^{\mathrm{a}}$, DaeWook Kim ${ }^{*} \mathrm{~b}$ \\ ${ }^{a}$ The Institute of Optics and Electronics, Chinese Academy of Sciences, Chengdu, China; ${ }^{b}$ College \\ of Optical Sciences, University of Arizona, Tucson, AZ 85721, USA
}

\begin{abstract}
Freeform optics provide excellent performance for a wide variety of applications. However, obtaining an accurate freeform surface measurement is highly challenging due to its large aspheric/freeform departure. It has been proven that SCOTS (Software Configurable Optical Test System), an advanced deflectometry system developed at the University of Arizona, can measure the departure of a freeform surface from the desired shape with nanometer accuracy. Here, a new data processing technique was used to measure a freeform surface without any prior knowledge of the shape of the surface. Knowing only the geometry of one point on the test surface, this method can take a blind measurement of a freeform surface and arrive at the true surface through iterative construction.
\end{abstract}

Keywords: Deflectometry, iterative, blind, freeform, metrology, SCOTS

\section{INTRODUCTION}

As the performance demand of optics increases, the use of freeform surface design has become increasingly more common. Freeform surfaces allow for excellent optical performance in a variety of applications. One major challenge in the process of fabricating freeform surfaces is obtaining accurate surface profiles [1]. There are a variety of methods for measuring reflective surface profiles, including using a coordinate measuring machine (CMM), a contact-type profilometer, or a computer generated hologram (CGH) interferometric test [2]. Additionally, it has been shown that a phase measuring deflectometry (PMD) system is able to obtain accurate surface profiles with high dynamic range [3-5]. This paper deals with implementing a next generation data processingtechnique to the software configurable test system (SCOTS), which is a PMD device.

The SCOTS system consists of a source which displays phase-shifting fringes, a unit under test (UUT), and a camera. The test can be thought of as a reverse Shack-Hartmann test, in which rays are traced from the camera off of the mirror and back to the source screen $[6,7]$. The SCOTS device has been applied to metrology of freeform surfaces with nanometer level accuracy, with results comparable to interferometric testing $[8,9]$. To date, SCOTS has been dependent upon a theoretical model of the UUT surface to perform a comparative ray trace in order to obtain high accuracy slope data [10]. This represents a practical limitation to the SCOTS system, as an arbitrary optic can only be measured when a theoretical surface map is provided.

In this study we investigated an iterative surface reconstruction method in which the software converges upon the surface profile of the UUT, with no prior knowledge (i.e. blind) of the UUT. In other words, the deflectometry data processing software does not require any information describing the UUT's surface shape (such as radius of curvature, conic constant, etc...). We describe the iterative surface reconstruction approach and how it was implemented in Section 2. In Section 3, the experimental setup used to demonstrate the blind deflectometry system is described. The preliminary results of the investigation and the performance of the system aredescribed in Section 4, followed by concluding remarks in Section 5.

*1etter2dwk@hotmail.com

AOMATT 2016: Optical Test, Measurement Technology, and Equipment, edited by

Yudong Zhang, Fan Wu, Ming Xu, Sandy To, Proc. of SPIE Vol. 9684, 96843X

(c) 2016 SPIE CCC code: 0277-786X/16/\$18 - doi: 10.1117/12.2243264

Proc. of SPIE Vol. $968496843 X-1$ 


\section{METHODOLOGY}

\subsection{Iterative Surface Construction}

We developed a software package which allowed for iterative surface construction of the UUT which was incorporated into the deflectometry software processing pipeline.The data processing algorithm operates by taking one known position on the mirror and making the starting assumption of a flat mirror at that location. This flat is the theoretical surface in the first iteration.

A ray trace calculation is then performed using the measured deflectometry data and the UUTevaluated surface shape is determined. The difference between the evaluated surface and the theoretical surface is calculated.Thisdiscrepancy information is fed back into the software and used to adjustthe theoretical surface in the next iteration. The comparison, adjustment, and new surface evaluation is repeated. This iterative process, started from a blind guess (i.e. flat surface), continues until the evaluated and theoretical model converge. At this point the evaluated surface is reported as the UUT's final surface shape.The concept of this iterative surface reconstruction process is presented in Figure 1.

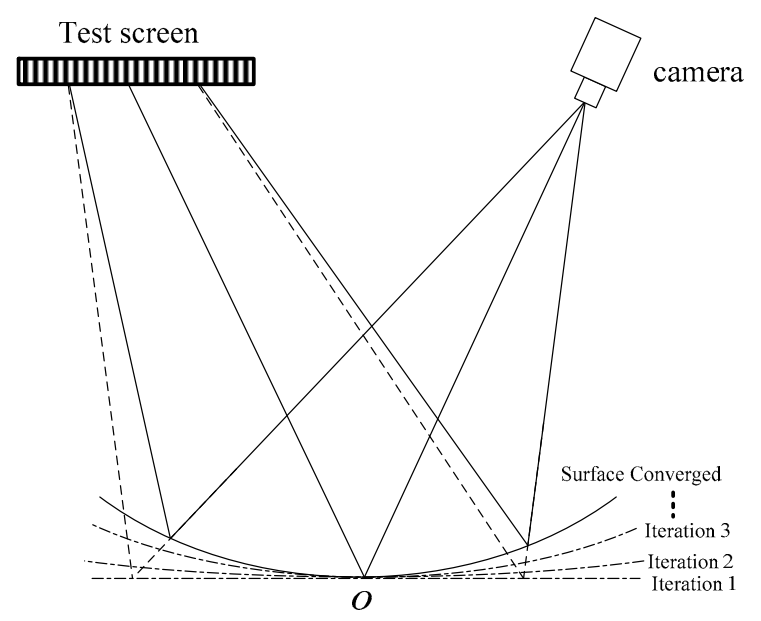

Figure 1. The blind deflectometry data processing concept of iterating through surface shapes until the results converge to a final reconstructed surface ( $O$ represents a known point on the optical surface).

In order for the iterative surface reconstruction to work, accurate knowledge of one point ( $O$ in Figure 1$)$ on the UUT must be known, as this is the seed point from which the surface is constructed in the algorithm. Additionally, in order to trace rays, knowledge of the ray path from camera to UUT to screen must be known. The methods taken to obtain the experimental data are described in Section 2.2.

\subsection{System Calibration for Iterative Surface Construction}

To obtain the necessary information for the iterative surface reconstruction a simple calibration method was developed. This process allowed for mapping ray paths in the blind deflectometry system, as well as obtaining geometrical position information of the UUT and the screen.

To map the ray path in the configuration, a calibration screen displayingpoints with a Gaussian profile was set up at the first position (Cali pos 1 in Figure 2) in front of the camera and an image was recorded. The physical location of theGaussian points on the screen were measured using a Point Source Microscope (PSM) device attached to a CMM, following a similar procedure used for previous SCOTS calibration [11]. This represents one space in which geometry was defined, referred to asCMM space. A phase shifted sinusoidal pattern was then displayed, and the location of all pixels on the screen was calculated (in the same CMM space). The screen was then moved axially to the second position(Cali pos 2 in Figure 2) and theidentical calibration procedure was repeated. From these two-plane calibration 
data setsthe ray path mapping from the camera was determined. The schematic system calibration process is demonstrated in Figure 2.

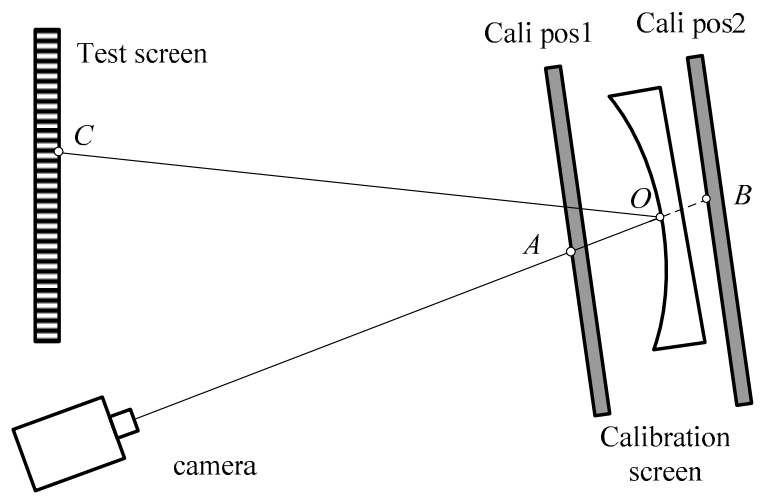

Figure 2.The schematic layout for the blind deflectometry system calibration procedure. A calibration screen was moved from calibration position 1 (Cali pos 1) to calibration position 2 (Cali pos 2) and data was recorded from each position. By recording the position of point $\mathrm{A}$ and $\mathrm{B}$ for a single ray, a unique ray path isdetermined. Positions $O$ and $C$ were then measured to correlate the ray path from the camera to the UUT to the test screen.

The position of the test screen is precisely measured using the PSM and CMM.With the ray mapping calibrated for the system, the UUT is placed between the first plane (Cali pos 1) and the second plane (Cali pos 2), as shown in Figure 2. Finally, the actual seed position $(O)$ on the UUT was measured by the PSM (in the same CMM space).

\section{EXPERIMENTAL SETUP}

To demonstrate the concept of the iterative surface reconstruction algorithm a blind measurement was prepared and performed.In order to provide independent interferometric comparisondata, a $50 \mathrm{~mm}$ (in diameter) spherical mirrorwith a radius of curvature of $200 \mathrm{~mm}$ fabricated by asingle-point diamond turning machine was selected as the UUT. The maximum sag of the mirror surface is about $1.57 \mathrm{~mm}$. The calibration procedure described in Section 2.2 was followed to prepare the experimental set-up configuration shown in Figure 3. The UUT was placed in the system and the captured deflectometry images were collected. The measured data was processed using the iterative surface reconstruction algorithm. Once again, no knowledge of the theoretical (or expected) surface shape of UUT was provided to the data processing software package for the measurement. The data processing converged upon a reconstructed final surface, which was defined inCMM coordinates. Thesurfacemetrology data was then transferred to the UUT mirror coordinates to represent the surface figure map.

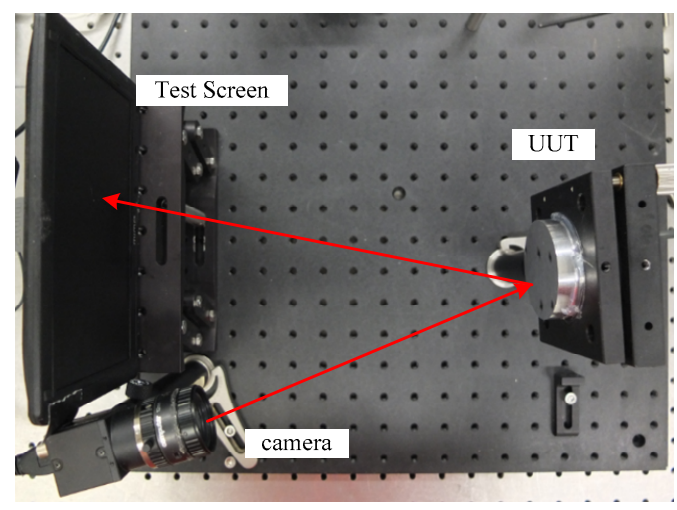

Figure 3.Experimental setup used for the iterative blind deflectometry test. 
To estimate the accuracy of the iterative solution a spherical fit was applied to the final measurement data. The difference in terms of radius curvature between the measured value and the givenspecification value was compared. For a more quantitative assessment of the blind approach, the measured surface map was analyzed using Zernike terms and compared against interferometric surface testing data. A comparison was made as a function of removed Zernike terms and the preliminary resultsare presented in Section 4.

\section{PRELIMINARY EXPERIMENTAL RESULTS}

\subsection{Iteratively Reconstructed Surface Map}

Figure 4shows the iteratively reconstructed surface map generated by the blind deflectometry measurement ofthe diamond turned spherical mirror. The spherical fitting resulted in a radius of curvature value of $200.034 \mathrm{~mm}$. The manufacturer specified the diamond turned mirror to be a spherical mirror with a radius of curvature of $200.000 \mathrm{~mm}$, resulting in a difference of 34 microns for the radius of curvature.
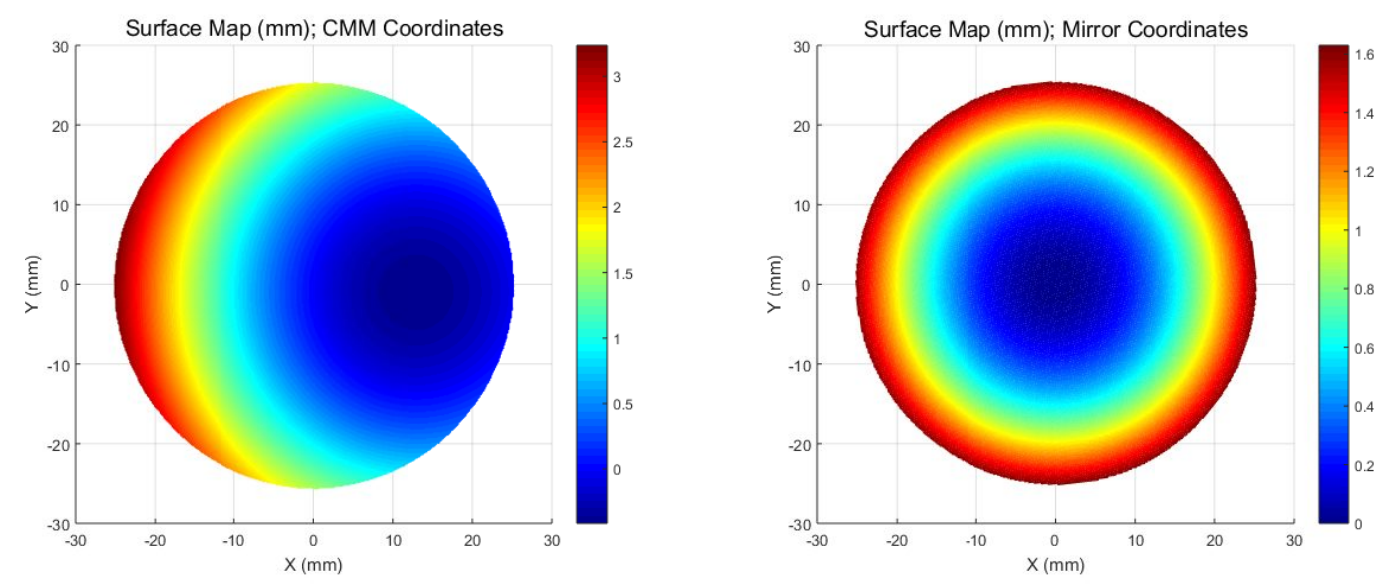

Figure 4.The reconstructed surface map by iterative deflectometry data processing in the original CMM coordinates (left), and in themirror coordinates centered at the UUT's vertex (right).

\subsection{Comparison between Interferometry and Deflectometry Results}

A comparison between interferometry and deflectometry data has been made for a quantitative assessment based on Zernike terms. The preliminary comparison results are presented in Figure 5 for different Zernike terms subtracted.

The residual root mean square (RMS)surface deviation of the reconstructed surfaceafter subtracting the best fit sphere (radius of curvature: $200.034 \mathrm{~mm}$ ) was $0.14 \mu \mathrm{m}$ (upper left in Figure 5). An interferometric measurement (using a Zygo VeriFire ATZ Interferometer) of the same mirror surface produced a residual RMS value of $0.15 \mu \mathrm{m}$ (lower left in Figure 5). When Zernike term 1-6 (i.e. Piston, Tip-tilt, Power and Astigmatism) were removed, the blind deflectometry'sresidual RMS was $0.07 \mu \mathrm{m}$ (upper right in Figure 5), and the interferometric data reported anRMS value of $0.05 \mu \mathrm{m}$ (lower right in Figure 5).

Although the overall RMS values matches within about $+/-0.02 \mu \mathrm{m}$, the actual shape of the surface figure shows some differences. For instance, the maps (right column in Figure 5) after subtracting Zernike term 1-6 include some coma-like differences. The difference could be due to the uncertainty of the deflectometry system or some other effects. However, the concept of the blind deflectometry data processing has been proven from these promising preliminary results. 


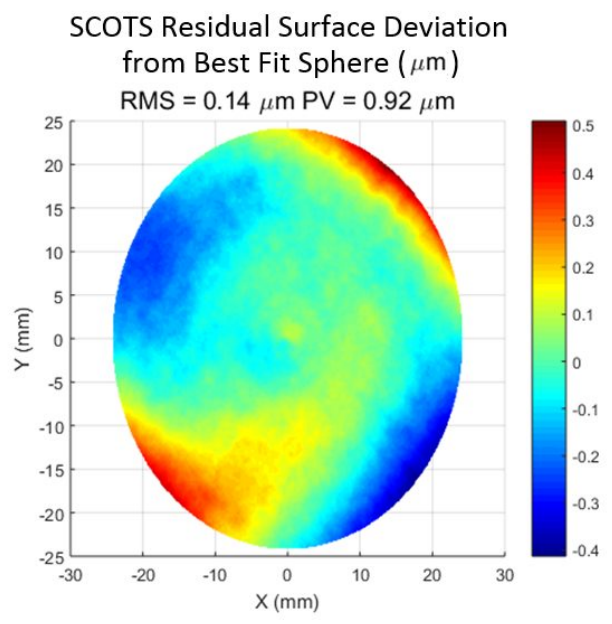

Interferometer Residual Surface Deviation from Best Fit Sphere $(\mu \mathrm{m})$

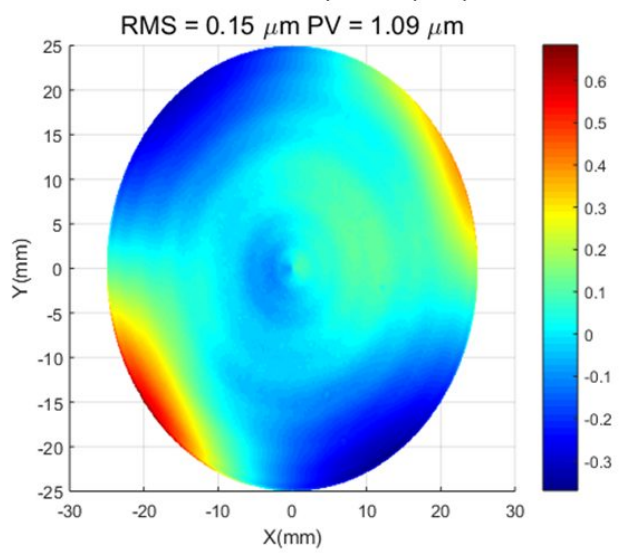

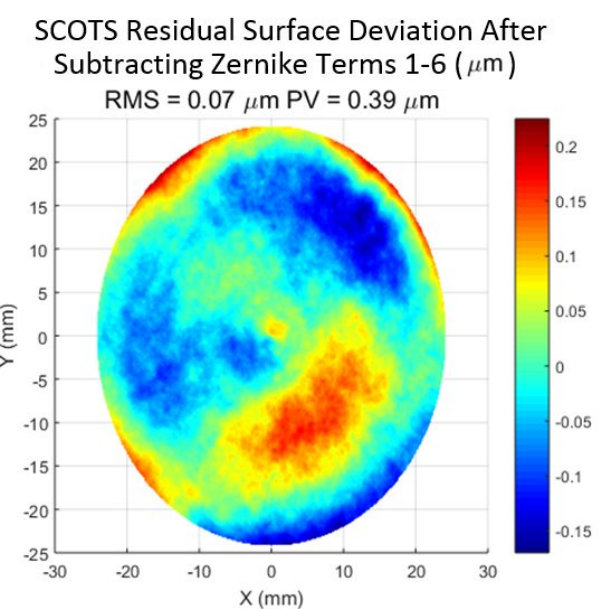

Interferometer Residual Surface Deviation After Subtracting Zernike Terms 1-6 $(\mu \mathrm{m})$

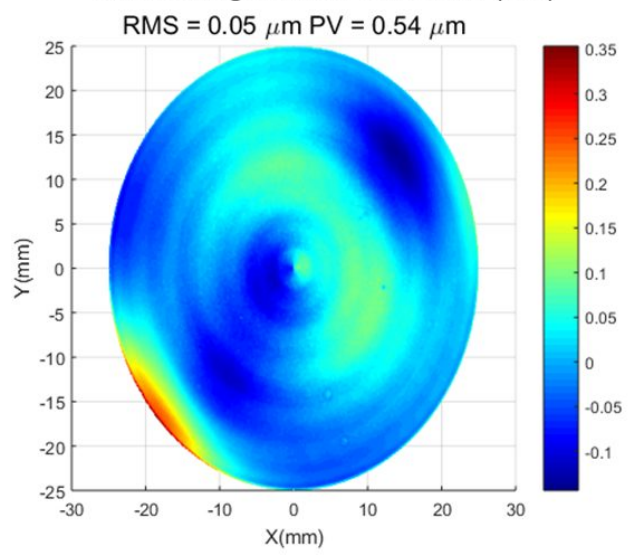

Figure 5. Comparison between the iteratively reconstructed surface map using the blind deflectometry setup (upper row) and the interferometric surface measurement data using a Zygo VeriFire ATZ Interferometer (lower row). The left column presents the surface map after subtracting the best fit sphere (or Zernike 1-4 terms) and the right column shows the surface maps after subtracting Zernike $1-6$ terms (i.e. Piston, Tip-tilt, Power and Astigmatism).

\section{CONCLUDING REMARKS}

The iteratively reconstructed surface data produced by the blind deflectometry setup demonstrated a measurement of optical surface with sub-micron RMS surface deviation features. When compared to interferometric results, the RMS values are comparable for different Zernike term removal cases. This preliminary result shows a promising possibility that the blind surface reconstruction algorithm might provide a reliable and accurate metrology solution without requiring measured surface shape information as an input.A follow-up paper with more systematic experimental data and comprehensive description/analysis will be presented in a separate publication.By removing the requirement of prior knowledge of the UUT for an accurate freeform/aspheric surface measurement, blind deflectometry can extendits capabilities greatly and could be able to take more versatile measurements while maintaining the same high performance and large dynamic range that PMD offers. 


\section{ACKNOWLEDGMENT}

We are grateful for support from the Youth Innovation Promotion Association,CAS (2016339). Additionally, we would like to thank Dr. Yuankun Liu, Sichuan University, for the helpful discussions. We also would like to thank the II-VI Foundation, which funded the software and system development through the II-VI Foundation Block Grant.

\section{REFERENCES}

[1] Fang, F.Z., Zhang, X.D., Weckenmann, A., Zhang, G.X., Evans, C., "Manufacturing and measurement of freeform optics," CIRP Annals - Manufacturing Technology 62(2013), 823-846 (2013).

[2] Valente, M., Lewis, B., Melena, N., Smith, M., Burge, J. H., Zhao, C., "Advanced surface metrology for meterclass optics," Proc. SPIE 8838, 88380F (2013).

[3] Knauer, M. C., Kaminski, J., Hausler, G., "Phase measuring deflectometry: a new approach to measure specularfree-form surfaces," Proc. SPIE 5457, 366 (2004).

[4] Li, W., Sander, M., Geslerich, A., Burke, J., “Absolute optical surface measurement with deflectometry," Proc. SPIE 8494, 84940G (2013).

[5] Faber, C., Olesch, E., Krobot, R., Häusler, G., "Deflectometry challenges interferometry: the competition gets tougher!" Proc. SPIE 8493, 84930R (2012).

[6] Su, P., Parks, R. E., Wang, L., et al., "Software configurable optical test system: a computerized reverse Hartmanntest" Applied Optics 49(23), 4404-4412 (2010).

[7] Su, P., Wang, S., Khreishi, M., Wang, Y., et al., "SCOTS: a reverse Hartmann test with high dynamic range forGiant Magellan Telescope primary mirror segments," Proc. SPIE 8450, 84500W (2012).

[8] Huang, R., Su, P., Burge, J.H., Huang, L., Idir, M.,"High-accuracy aspheric x-ray mirror metrology using Software Configurable Optical Test System/deflectometry" Optical Engineering 54(8), 084103 (2015).

[9] Huang, R., Su, P., Horne, T., Zappellini, G., Burge, J., "Measurement of a large deformable aspherical mirror using SCOTS (Software Configurable Optical Test System)" Proc. SPIE 8838, 883807(2013).

[10] Su, P., Khreishi, M., Huang, R., Su, T., Burge, J., "Precision aspheric optics testing with SCOTS: a deflectometery approach" Proc. SPIE 8788, 87881E (2013).

[11] Huang, R., Su, P., Burge, J., "Deflectometry measurement of Daniel K. Inouye Solar Telescope primary mirror" Proc. SPIE 9575, 957515(2015). 